

\title{
Propriétés arithmétiques des suites récurrentes linéaires
}

\author{
Par M. Mignotte
}

\section{Introduction .}

Les suites récurrentes linéaires interviennent dans de nombreux domaines. Elles sont d'abord apparues en combinatoire avec Fibonacci [ Fi ], dès 1202, pour modéliser l'évolution d'une population de lapins ( en l'absence de prédateur ); on trouve des exemples de dénombrements liés aux suites récurrentes linéaires dans la plupart des ouvrages de combinatoire, par exemple dans le livre de Comtet [ $\mathrm{Co}$ ]. Dans le domaine de l'algèbre, elles sont liées à diverses questions d'élimination, cet aspect est présenté en particulier en [C.M.P.] : un autre point de vue, lié aux fonctions symétriques ( voir [ $\mathrm{Mac}$ ]), est donné par A. Lascoux, [ Las ]. Elles apparaissent en théorie des langages : morphismes itérés sur un monoïde ( DOL-systèmes ..., voir le livre de J. Berstel [ Ber1 ]), langages rationnels ( voir [ B.R.] ). Elles constituent un objet fondamental en théorie des communications où l'étude des suites récurrentes à valeurs dans un corps fini équivaut à celle des registres à décalage (en anglais, shift registers ) : voir les références [Go ], [L.N.], [ McW.Sl. ], [Rue ], [ Se ], [ $\mathrm{Z}$ ] ainsi que le cours tout récent de D. Ferrand [ $\mathrm{Fe}$ ] qui donne un point de vue mathématique, à la fois concis et clair, de cette théorie. On les rencontre bien sûr aussi dans l'étude des équations aux différences finies : voir les livres de Gel'fond [ $\mathrm{Ge}$ ], Milne-Thomson [M-Th. ] et P. Montel [ Mo], ainsi que le chapitre 7 du livre de Henrici, [ He ] . Elles sont aussi utiles en logique, approximation, pour la production de suités pseudo-aléatoires (à ce sujet voir Knuth, $[\mathrm{Kn}]$ ) ... 
Cet article ne traite que des propriétés arithmétiques des suites récurrentes linéaircs. Le véritable créateur de ce domaine est Edouard Lucas, cf. son ouvrage [ Lu ] . Cette question a donné lieu ensuite à de très nombreux travaux et constitue déjà tout un chapitre du livre de Dickson, [ Di ], sur l'histoire de la théorie des nombres. Voir aussi un important travail de Carmichael en 1920, [ Car ]. Une étude générale des suites récurrentes linéaires figure dans le cours de Pisot [ $\mathrm{Pi}]$, où sont présentés quelques aspects arithmétiques de ces suites, les aspects liés à l'étude de certains ensembles d'entiers algébriques.

Les propriétés arithmétiques des suites récurrentes linéaires qui sont plus particulièrement étudiées ici ont fait l'objet de nombreux travaux durant ces demières années. Les principaux problèmes considérés sont :

- étude des zéros d'une suite donnée : [ B.M. ], [ Mi2 ] ,...

- minoration du terme général : [ Ma ], [ Mil ], [ M.S.T.] ,...

- bornes pour les multiplicités : [ Beu ], [ Ku ].

- minoration de $P\left(u_{n}\right)$, le plus grand diviseur premier de $u_{n}:[$ Ste2 ], [ Sho1 ] ...

- étude des répétitions : [ P.Sh ], [ Mi3 ],

- questions de rationalité : [ A ], [ Dw ], [ Bert ], [ Pa ], [ Can1 ], [U], [Poo1] .

Les outils utilisés dans ces travaux sont :

- congruences et équation de Pell - Fermat : par exemple dans [Mi4] et [Mi5 ],

- analyse p-adique : e. g. dans [ $\mathrm{Ku}]$ et [ $\mathrm{Mi2}$ ],

- fonctions hypergéometriques : e.g. dans [B.T.],

- formes linéaires de logarithmes : e. g. dans [Mi1 ], [M.S.T.], [ Mi7 ], ...

- théorèmes sur l'approximation diophantienne des nombres algébriques : le théorème de Roth - Ridout dans [Ma], celui de Roth - Schmidt - Schlickewci en [Ev ], [ Lau1], [ Sch.vdP. ] ,... .

Parmi ces outils, le scul qui conduise automatiquement à des résultats " effectifs " est la théorie des formes linéaires de logarithmes. 
On trouve des informations récentes sur les thèmes abordés dans cet exposé dans les références suivantes : la seconde partie de l'article de synthèse [ C.M.P.] porte sur l'étude arithmétique des suites récurrentes linéaires ; c'est aussi le thème de plusieurs chapitres de l'ouvrage de T. Shorey et R. Tijdeman [S.T.] ; l'article [ Poo2] de van der Poorten contient aussi une présentation originale de plusieurs problèmes arithmétiques célc̀bres sur les suites récurrentes linéaires.

Le présent texte a été conçu comme un complément à [ C.M.P. ] et insiste sur les progrès réalisés depuis la rédaction de cet article; une partie des informations données ici provient de [S.T.] et [ $\mathrm{Poo} 2$ ], qui sont tous deux postérieurs à [C.M.P.] . L'article de synthèse [ E.G.S.T. ] présente aussi un panorama des progrès récents sur les suites récurrentes linéaires.

La plupart des problèmes sont bien plus faciles pour les suites binaires que pour les suites ternaires (ou d'ordre plus élevé !). En fait, plusieurs problèmes sur les suites récurrentes ternaires restent ouverts : par exemple le nombre maximal possible de zćros d'une suite récurrente linéaire cubique à valeurs entières. De plus, beaucoup de travaux sur les suites binaires portent sur des questions très spéciales, qui ne se généralisent pas au cas des suites d'ordre quelconque ; ces suites font l'objet des chapitres 2 et 3 de [S.T.], elles ne seront pas étudiées en détail ici.

Parmi les suites récurrents linéaires cubiques à valeurs entières, la suite de Berstel $\left(b_{n}\right)$, qui est définie par $b_{0}=0, b_{1}=0, b_{2}=1, b_{n}=2 b_{n-1}-4 b_{n-2}+4 b_{n-3}$ pour $n \geq 3$, semble jouer un rôle très particulier.

D'abord, c'est le seul exemple connu d'une suite linéaire récurrente ternaire nondégénérée qui possède six zéros ( une suite récurrente linéaire est non-dégénérée ne possède qu'un nombre fini de zéros), il a été démontré en [Des] qu'il s'agit de la seule suite, au scin d'une vaste classe de suites récurrentes ternaires, à posséder autant de zéros, de plus, 
dans le travail non publié [ Beu2 ], Beukers a démontré qu'une suite linéaire récurrente ternaire non-dégénérée à valeurs entières possède au plus sept zéros.

Deuxièmement, la suite de Berstel contient beaucoup de répétitions, en fait on a montré en [Mi3] que l'équation $u_{m}= \pm u_{n}$, pour des indices $m, n \in Z$, possède exactement 21 solutions $(m, n)$ avec $m<n$, ces solutions étant explicitement calculées.

En [ M.T. ], on étudie l'équation $u_{n}= \pm 2 r 3^{s}, r$ et $s \in Z$, et là encore il s'avère que la suite de Berstel semble constituer un exemple remarquable : cette équation possède exactement 44 solutions, pour $\mathrm{n}$ parcourant $\mathrm{Z}$.

Ces deux derniers travaux présentent aussi l'intérêt de résoudre complètement des équations pour lesquelles on ne connait actuellement aucune méthode algorithmique de résolution.

Le plan de cet article est le suivant :
I . Préliminaires
II . Etude des zéros d'une suite donnée
III . Minoration du terme général
IV . Bornes pour les multiplicités
V. Minoration de $P\left(u_{p}\right)$
VI . Etude des répétitions
VII . Questions de rationalité
Bibliographie . 


\section{I . Préliminaires .}

Les notations introduites dans ce paragraphe sont très proches de celles de [ C.M.P.] , afin de pouvoir renvoyer implicitement le lecteur à cet article pour des démonstrations et plus de détails ; elles seront conservées dans toute la suite.

On se place dans un corps $K$ ( commutatif). Une suite récurrente linéaire $\left(u_{n}\right)$ sur $K$ est une suite qui vérifie une relation du type suivant

$$
u_{n+m}=a_{m-1} u_{n+m-1}+\ldots+a_{0} u_{n}, n=0,1,2, \ldots, \text { avec } a_{0} \neq 0,
$$

où $\mathrm{m}$ est un entier et $a_{m-1}, \ldots, a_{0}$ des éléments de $K$. Une telle suite est complètement déterminée par ses $m$ premières valeurs $u_{0}, \ldots, u_{m-1}$. On dit que la suite est d'ordre $m$. Lorsque $m=2$, la récurrence est dite binaire. Une suite récurrente d'ordre trois sera dite ternaire ou encore cubique.

Le polynôme compagnon d'une suite qui vérifie (1) est par définition égal à

$$
G(X)=X^{m}-a_{m-1} X^{m-1}-\ldots-a_{1} X-a_{0},
$$

on notera ses différentes racines par $\omega_{1}, \ldots, \omega_{k}$, la multiplicité de la racine $\omega_{1}$ étant égale à $\tau_{\mathrm{i}}$, pour $\mathrm{i}=1,2, \ldots, \mathrm{k}$. Par commodité, ces $\omega_{\mathrm{i}}$ sont souvent appelées les racines de la suite $\left(u_{n}\right)$.

Considérons la série génératrice de la suite $\left(\mathrm{u}_{\mathrm{n}}\right)$ :

$$
U(X):=\sum_{n=0}^{\infty} u_{n} X^{n}
$$

Soit $B(X)$ le polynôme réciproque de $G(X)$, i. e. $B(X)=1-a_{m-1} X-\ldots-a_{0} X^{m}$. Posons $A(X)=U(X) . B(X)$. Grâce à la formule (1), il vient

$$
A(X)=\sum_{j=0}^{m}\left(u_{j}-\sum_{i=1}^{j} a_{m-1-i} \cdot u_{j-i}\right) X^{j}
$$


Ainsi, A ( X ) est un polynôme et la formule

$$
U(X)=\frac{A(X)}{B(X)}
$$

montre que la série formelle $\mathrm{U}(\mathrm{X})$ est une série rationnelle. Dans le cas particulier où la suite $\left(u_{n}\right)$ est à valeurs entières, on voit que les polynômes $A$ et $B$ définis ci-dessus sont à coefficients entiers, avec $\mathrm{B}(0)=1$, résultat dû à $\mathrm{P}$. Fatou , $[\mathrm{Fa}]$.

Notons aussi la formule (voir [ Poo2 ] § 2.6)

(4) $\frac{1}{B(X)}=\sum_{n=0}^{\infty} \sum_{j_{1}+2 j_{2}+\ldots+m j_{m}=n} \frac{\left(j_{1}+\ldots+j_{m}\right) !}{j_{1} ! \ldots j_{m} !} a_{m-1} j_{1} \ldots a_{0} j_{m} \quad X^{n}$.

En développant la fraction rationnelle (3) en éléments simples, on constate que le terme général de la suite $u_{n}$ est donné par une expression de la forme

$$
u_{n}=\sum_{i=1}^{k} P_{i}(n) \omega_{i}^{n}, \text { avec } P_{i}(n)=\sum_{j=1}^{\tau_{i}} \beta_{i j}\left(\begin{array}{c}
n+j-1 \\
j-1
\end{array}\right)
$$

où les $\beta_{\mathrm{ij}}$ appartiennent au corps $K\left(\omega_{1}, \ldots, \omega_{\mathrm{k}}\right)$; une expression telle que (5) sera appelée un polynôme exponentiel. On supposera que cette expression ne comporte pas de termes triviaux, c'est à dire que pour chaque indice i il existe au moins un des coefficients $\beta_{\mathrm{ij}}$ qui n'est pas nul. Dans le cas où le corps $K$ est de caractéristique nulle, les $\mathrm{P}_{\mathrm{j}}$ sont des polynômes non nuls, à coefficients dans le corps $K\left(\omega_{1}, \ldots, \omega_{\mathbf{K}}\right)$, et dont les degrés vérifient la majoration $\operatorname{deg}\left(P_{i}\right)<\tau_{j}$, pour $i=1,2, \ldots, k$.

La formule (5) est très utile, elle servira systématiquement dans la suite. C'est grâce à elle que l'on peut appliquer l'analyse réelle, l'analyse p-adique, la théorie des formes linéaires de logarithmes et le théorème d'approximation de Roth-Ridout-Schmidt-Schlikewei aux suites récurrentes linéaires à valeurs algébriques.

Enfin, on vérifie qu'une suite $\left(\mathrm{u}_{\mathrm{n}}\right)$ à valeurs dans un corps $K$ et donnée par (5) vérifie une relation de la forme (1), où les $a_{\mathrm{j}}$ sont des éléments de $K$.

Nous avons donc montré que les définitions (1), (3) et (5) sont équivalentes. 
La caractérisation (3) montre que l'ensemble des suites récurrentes linéaires est stable par addition et par produit de Cauchy [i. e. $w_{n}=u_{n} v_{0}+u_{n-1} v_{1}+\ldots+u_{0} v_{n}$ ] ; tandis que la caractérisation (5) montre qu'il est stable par produit de Hadamard [ c'est à dire par l'opération définie par $w_{n}=u_{n} v_{n}$ ].

Exemple : L'exemple le plus populaire de suite récurrente linéaire et aussi sans doute lc plus ancien (il date de 1202) est la suite $\left(F_{n}\right)$ de Fibonacci définie par les conditions

$$
F_{0}=0, F_{1}=1, F_{n+2}=F_{n+1}+F_{n} \text { pour } n \geq 0 \text {, }
$$

de sorte que ses valeurs successives sont $0,1,1,2,3,5,8,13,21,34,55,89,144, \ldots$; dans ce cas la formule (5) s'écrit

$$
F_{n}=\frac{\omega_{1}{ }^{n}-\omega_{2}{ }^{n}}{\omega_{1}-\omega_{2}} \quad \text { où } \quad \omega_{1}=\frac{1+\sqrt{5}}{2} \quad \text { et } \quad \omega_{2}=\frac{1-\sqrt{5}}{2}
$$

La fonction $\mathrm{U}(\mathrm{X})$ définie en (2) est

$$
U(X)=\frac{X}{1-X-X^{2}}
$$

et enfin la formule (4) conduit à la relation

$$
F_{n+1}=\sum_{j=0}^{n}\left(\begin{array}{l}
n-j \\
j
\end{array}\right)
$$

Cette suite a suscité tellement d'études qu'il existe un journal périodique consacré à son étude et à des questions voisines, le Fibonacci Quaterly.

Remarque : La formule (1) permet d'étendre la définition de $u_{n}$ pour $\mathbf{n}$ négatif, si on pose $u_{n}^{\prime}=u_{-n}($ pour $n=0,1,2 \ldots)$, on a

$$
u_{n+k}^{\prime}=-\left(a_{k+1} / a_{k}\right) u_{n+k-1}^{\prime}-\ldots-\left(a_{1} / a_{k}\right) u_{n+1}^{\prime}+\left(1 / a_{k}\right) u_{n}^{\prime} \text {. }
$$

Parfois, nous considérerons donc la suite $u_{n}$ pour $n$ parcourant $\mathbf{Z}$ tout entier. 


\section{II . Etude des zéros d'une suite donnée.}

Un des théorèmes les plus remarquables sur les suites récurrentes linéaires concerne la nature arithmétique de l'ensemble $Z$ des zéros d'une suite récurrente $\left(u_{n}\right)$ (i.e. de l'ensemble des indices $n$ pour lesquels $u_{n}=0$ ); il s'agit du résultat suivant.

Théorème (Skolem - Mahler - Lech $)$. - Soit $\left(u_{n}\right)$ une suite récurrente linéaire à valcurs dans un corps de caractéristique nulle. Alors l'ensemble $Z$ des zéros de cette suite est égal à une union finie de progressions arithmétiques ( certaines de ces progressions pcuvent être de raison nulle et l'union peut même être vide !).

La méthode de démonstration, due à Skolem [ Sk ], constitue un exemple remarquable d'utilisation de l'analyse p-adique ( pour une introduction aux nombres p-adiques le lectcur pourra consulter Borevitch-Schafarevitch [ B.S.] , J.P. Serre [ Se ] ou Cassels [ Cas ], et pour une étude plus détaillée de l'analyse p-adique Y. Amice [ A ] , K. Mahler [ Ma3 ] ou Ch. Pisot [ Pi ]).

Dans le cas où la suite est à valeurs entières, en choisissant un nombre premier p convenable on démontre qu'il existe un entier $\mathrm{T}$ positif tel que chacune des $\mathrm{T}$ fonctions $f_{i}(n)=u_{n T+i}$ pour $i=0,1, \ldots, T-1$ s'étende en une fonction analytique sur l'ensemble $Z_{p}$ des entiers p-adiques. Du fait que $Z_{p}$ est un ensemble compact, chacune de ces fonctions $f_{i}$ ou bien ne possède qu'un nombre fini de zéros, ou bien est identiquement nulle. D'où le résultat dans ce cas.

Puis Mahler [ Ma1 ] a étendu ce résultat au cas où les $u_{n}$ sont des nombres algébriques. Enfin, Lech [ Le ] a démontré le cas général donné ci-dessus.

On peut noter que l'article de $\mathrm{Ph}$. Robba [ Ro ] fournit une majoration de la raison des progressions arithmétiques infinies contenues dans l'ensemble $Z$; pour un résultat plus général voir [P.R. ]. 
On déduit aisément du théorème la conséquence suivante $:$ si $\left(u_{n}\right)$ possède une infinité de zéros alors, dans la formule (5), pour chaque indice $i$ il existe un indice $j \neq i$ tel que le quotient $\omega_{\mathrm{i}} / \omega_{\mathrm{j}}$ soit une racine de l'unité. Ceci permet de démontrer ( $\mathrm{cf}$. [ $\mathrm{B} \mathrm{M}$ ] ) que le problème de savoir si $\left(u_{n}\right)$ possède ou non une infinité de zéros est décidable, en réponse à une question de M.P. Schützenberger.

On dira qu'un suite récurrente linéaire est non dégénérée si elle est non nulle et si, de plus, aucun des quotients $\omega_{\mathrm{i}} / \omega_{\mathrm{j}}$ n'est une racine de l'unité ; on vient juste de voir qu'une telle suite ne possède qu'un nombre fini de zéros.

Une autre question, autrement plus difficile, est la détermination explicite de l'ensemble $Z$ des zéros d'une suite récurrente linéaire non dégénérée donnée. On dispose alors essentiellement de deux méthodes : la méthode p-adique et la méthode de Baker, [ Ba ] . La première méthode consiste à appliquer un théorème de Strassmann ( [ Str ] , ce théorème est aussi démontré par exemple en [ Lew ] et [ Cas ]) qui fournit une majoration du nombre de zéros d'une fonction analytique p-adique non identiquement nulle, mais ce résultat ne permet de déterminer $Z$ que dans le cas où, par chance, la majoration de ce nombre de zéros est exactement égale au nombre de ces zéros. La seconde méthode - présentće au paragraphe suivant - ne s'applique que lorsque les $\omega_{i}$ de la formule (4) vérifient certaines hypothèses particulières, mais elle fournit alors un majorant explicite $M$ des éléments de $Z$ et ensuite " il suffit " de déterminer les $n<M$ tels que $u_{n}$ soit nul (comme en pratique $M$ est très grand, ceci demande un travail important et une technique possible est alors d'étudier la suite modulo de nombreux nombres premiers ). Ces deux méthodes ont été appliquées en [ Mi1] pour montrer que l'ensemble des zéros de la suite de Berstel est exactement égal à $Z=\{0,1,4,6,13,52\}$, résultat qui figure aussi dans [ Cas ] 
En [M.S.T.] , on démontre que si une suite récurrente linéaire non dégénérée à valeurs algébriques possède au plus quatre racines $\omega_{i}$ de module maximal alors on peut déterminer effectivement un entier $N$ tel que la relation $u_{n}=0$ implique $n<N$. De plus, Shorey et Tijdeman remarquent ( notes du chapitre 4 de [Sh.T. ] ) que la preuve de ce théorème ( qui figure aussi en [ Sh.T.], theorem 4.6) conduit au résultat suivant :

si $\left(u_{n}\right)$ est une suite récurrente linéaire non dégénérée à valeurs algébriques pour laquelle $k=5$ et $\left|\omega_{1}\right|=\left|\omega_{2}\right|=\ldots=\left|\omega_{5}\right|$ (rappelons que $k$ désigne le nombre de racines $\omega_{i}$, voir formule (5) ) alors la relation $u_{n}=0$ implique que $n$ est borné par un nombre calculable, ne dépendant que de la suite considérée.

Une extension du théorème de Skolem-Mahler-Lech aux germes de fonctions analytiques sur $\mathbf{C}$, solutions de certaines équations différentielles linéaires, a été donnće par J. P. Bézivin, [ Bez3 ] .

Terminons ce paragraphe par deux questions ouvertes :

- Problème : Existe-t-il un algorithme qui permette de calculer $Z$ pour toute suite récurrente linéaire à valeurs entières ?

- Conjecture : Il n'existe pas d'algorithme qui, étant données 21 suitcs récurrentes linéaires à valeurs entières $u^{(1)}, u^{(2)}, \ldots, u^{(21)}$, permette de savoir si l'équation

$$
u_{n_{1}}^{(1)}+u_{n_{2}}^{(2)}+\ldots+u_{n_{21}}^{(21)}=0
$$

possède ou non une solution $\left(n_{1}, n_{2}, \ldots, n_{21}\right) \in N^{21}$.

Il est à noter que cette conjecture est liée au problème de la possibilité de rendre effectif ou non le théorème d'approximation de Roth-Schmidt-Schlickewei. De manière précise, si cette conjecture est vraie alors ce théorème ne peut pas être rendu complètement effectif. 


\section{III . Minoration du terme général .}

Soit $\left(\mathrm{u}_{\mathrm{n}}\right)$ une suite récurrente linéaire à valeurs entières ( ou plus généralement à valeurs algébriques ), on veut minorer $\left|u_{n}\right|$ de manière non triviale pour $n$ assez grand.

Là encore, on utilise la formule (5). Dans le cas présent, les $\omega_{\mathrm{i}}$ sont donc des nombres algébriques complexes et les $P_{i}$ des polynômes ( non nuls) à coefficients algébriques complexes. On supposera de plus que la numérotation est telle que l'on a la suite d'inégalités $\left|\omega_{1}\right| \geq\left|\omega_{2}\right| \geq \ldots \geq\left|\omega_{k}\right|$.

Lorsqu'il existe une seule racine dominante, c'est à dire quand on a $\left|\omega_{1}\right|>\left|\omega_{2}\right|$, alors, trivialement, $\left|u_{n}\right|>\frac{1}{2} P_{1}(n) \omega_{1}^{n}$ pour $n \geq n_{0}$ (effectif).

Par contre, lorsque $\omega_{1}$ et $\omega_{2}$ sont de même module, avec $\omega_{1} \neq \pm \omega_{2}$, le problème se complique sérieusement: dans le cas le plus simple où $\mathrm{k}=2, \mathrm{P}_{1}=\mathrm{P}_{2}=1$ on voit que

$$
u_{n}=2 \rho^{n} \cos (n \theta) \text {, où on a posé } \omega_{1}=\rho e^{i \theta} \text {, avec } \rho \text { réel positif, }
$$

et minorer $\left|u_{n}\right|$ revient à minorer la quantité $|\Lambda|$, lorsque $\Lambda=n \theta-k \pi$ pour $n$ et $k$ parcourant $\mathrm{Z}$. Ainsi $\Lambda$ est une " forme linéaire de logarithmes de nombres algébriques ".

Les travaux de Gel'fond fournissent une minoration de $|\Lambda|$, ce qui a permis à $A$. Schinzel d'obtenir le premier, dans le cas des suites récurrentes linéaires binaires, une minoration effective du terme $\left|u_{n}\right|$. Grâce aux travaux de Baker, un théorème plus général a ćté obtenu en [ Mi2 ], puis ce résultat a été étendu en [M.S.T. ], où on démontre une minoration effective de $\left|u_{n}\right|$ lorsque le nombre $r$ maximum de nombres $\omega_{i}$ de module maximal est au plus trois et qu'aucun des quotients $\omega_{\mathrm{i}} / \omega_{\mathrm{j}}, 1 \leq \mathrm{i}<\mathrm{j} \leq \mathrm{r}$, n'est une racine de l'unité. Lorsque ce théorème s'applique on peut donc déterminer effectivement toutes les solutions de l'équation $u_{n}=a$, pour a fixé (et en particulier pour $a=0$, ce qui permet de traiter de cette manière la suite de Berstel ). 
Depuis peu, van der Poorten et Schlikewei ont démontré la minoration suivante : soit $\left(u_{n}\right)$ une suite récurrente linéaire non dégénérée à valeurs algébriques alors, pour tout nombre réel $\varepsilon>0$ fixé, il existe un entier no tel que l'on ait $\left|u_{n}\right| \geq\left|\omega_{1}\right|^{n(1-\varepsilon)}$ pour tout indice $n>n_{0}$ ( remarquer que cette estimation est essentiellement la meilleure possible puisque l'on a trivialement $\left|u_{n}\right|<\left|\omega_{1}\right|^{n(1+\varepsilon)}$ pour tout $n$ suffisamment grand). Leur démonstration utilise le théorème fondamental des S-unités, lui-même déduit du thćorème de Thue-Siegel-Roth-Schmidt-Schlikewei. Par conséquent ce résultat est actuellement ineffectif. Le théorème fondamental des S-unités est l'énoncé suivant :

Théorème fondamental des S-unités . - Soit $K$ un corps de nombres et soient $G$ un sous-groupe de type fini du groupe multiplicatif $K^{*}$ et $m$ un entier $\geq 2$. L'équation

$$
\mathrm{X}_{1}+\ldots+\mathrm{X}_{\mathrm{m}}=1
$$

n'a qu'un nombre fini de solutions $X_{1}, \ldots, X_{m} \in G$ telles qu'aucune somme particlle

$$
\mathrm{x}_{\mathrm{i}_{1}}+\ldots+\mathrm{X}_{\mathrm{i}_{\mathrm{t}}},\left(1 \leq \mathrm{i}_{1}<\ldots<\mathrm{i}_{\mathrm{t}} \leq \mathrm{m}\right)
$$

ne s'annulle.

Le lecteur trouvera des résultats partiels mais instructifs, ainsi que plusieurs conjectures très fortes, dans le travail [ L.P. ] de Loxton et van der Poorten, qui date d'il y a une dizaine d'années. On notera que la conjecture de cet article concernant une minoration " effective " de $\left|u_{n}\right|$ témoigne d'un sentiment opposé à la conjecture qui figure ici au paragraphe II , qui - elle - afirme qu'il existe des problèmes indécidables liés aux suites récurrentes linéaires. 
IV . Bornes pour les multiplicités.

Ce sujet a été traité en détail dans l'exposé [ Ti ] de R. Tijdeman, je le traiterai donc rapidement.

Nous considérons une suite récurrente linéaire $\left(u_{n}\right)$ à valeurs complexes. Pour un nombre complexe donné a, la a-multiplicité de cette suite est le nombre de solutions de l'équation $u_{n}=a$, tandis que la multiplicité de cette suite est le maximum de ses a-multiplicités ( on ne considère bien sûr que le cas où les a-multiplicités sont toutes finies, ce qui est vrai lorsque la suite est non dégénéré ).

La multiplicité des suites récurrentes binaires à valcurs entières non dégénérées a été très étudiée par M. Ward dans les années trente. De nombreux auteurs ont ensuite travaillé sur cette question : Skolem, Chowla, Lewis, Laxton, ... Enfin, Kubota [ $\mathrm{Ku}$ ] a démontré que la multiplicité d'une telle suite n'excède jamais 4 . Ce résultat a été ensuite amélioré par F. Beukers qui a montré que pour une telle suite l'équation $u_{n}= \pm$ a possède au plus trois solutions, sauf dans les cinq cas suivants :

$$
\begin{array}{llll}
u_{0}=u_{1}=1 \text { et } u_{n+2}=u_{n+1}-2 u_{n} & \text { où } & u_{0}=u_{1}=1, u_{2}=u_{4}=u_{12}=-1, \\
u_{0}=1, u_{1}=-1 \text { et } u_{n+2}=u_{n+1}-2 u_{n} & \text { où } & u_{0}=1, u_{1}=u_{3}=u_{11}=-1, \\
u_{0}=u_{1}=1 \text { et } u_{n+2}=3 u_{n+1}-4 u_{n} & \text { où } & u_{0}=u_{1}=1, u_{2}=u_{6}=-1, \\
u_{0}=u_{1}=1 \text { et } u_{n+2}=2 u_{n+1}-3 u_{n} & \text { où } & u_{0}=u_{1}=u_{5}=1, u_{2}=-1, \\
u_{0}=1, u_{1}=-1 \text { et } u_{n+2}=u_{n+1}+u_{n} & \text { où } & u_{0}=1, u_{1}=u_{3}=u_{4}=-1
\end{array}
$$

Les preuves de ces deux résultats reposent sur l'analyse p-adique.

Beukers et Tijdeman ont obtenu des résultats généraux sur la multiplicité des suites récurrentes linéaires binaires à valeurs complexes, voir [B.T.], ils utilisent des polynômes hypergéométriques, méthode introduite ( dans un contexte un peu différent) par Thue ct Siegel. 
Comme on l'a dit au $\S \mathrm{I}$, le problème de la multiplicité des suites récurrentes linéaires cubiques à valeurs entières n'est pas encore complètement résolu. Des résultats importants ont été démontrés par Beukers [ Beu2 ] et par B. Deshommes [ Des ]; voir à ce sujet la remarque de van der Poorten [ Poo2 ] , § 5.3.7 .

On peut utiliser l'analyse réelle dans la situation suivante : soit $U(x)$ un polynôme exponentiel de la forme $U(\mathrm{x})=\sum \mathrm{P}_{\mathrm{i}}(\mathrm{x}) \alpha_{\mathrm{i}} \mathrm{x}$, où les $\alpha_{\mathrm{i}}$ sont réels et positifs, alors $U$ possède au plus trois zéros réels. C'est le problème 75 du célèbre livre de Pólya et Szegö [ P.S. ] : on applique le théorème de Rolle. Il en résultc aussitôt qu'une suite récurrente linéaire d'ordre $\mathrm{m}$ dont les racines sont réelles et positives possède au plus $\mathrm{m}$ - 1 zéros et a une multiplicité bornée par $\mathrm{m}$, et aussi que si on suppose seulement les $\alpha_{i}$ réels alors une telle suite $u_{n}$ comporte au plus $2 \mathrm{~m}$ - 2 zéros ( on suppose que la suite comporte au moins $2 \mathrm{~m}-1$ zéros et on considère séparément les deux suites $u_{2 n}$ et $u_{2 n-1}$, l'une d'entre elles possède au moins $\mathrm{m}$ zéros, d'où une contradiction d'après le résultat précédent ). Dans le cas d'une suite récurrente linéaire cubique à valeurs réelles dont le polynôme minimal a trois racines simples, toutes réelles, en utilisant aussi l'analyse réclle Smiley $[\mathrm{Sm}]$ a démontré que l'équation $\mathrm{u}_{\mathrm{n}}=0$ possède au plus trois solutions, résultat retrouvé ensuite par Scott [Sc] et Picon [Pic] .

Du théorème fondamental des S-unités, Glass, Loxton et van der Poorten on déduit que dans le cas général d'une suite récurrente linéaire $\left(u_{n}\right)$ non dégénérée et à valeurs algébriques le nombre total de multiplicités (i. e. le nombre de solutions de $u_{m}=u_{n}$ avec $m<n)$ est fini. Il en résulte aussitôt que, pour tout a, la a-multiplicité de $\left(u_{n}\right)$ est finie ( résultat qui est aussi une conséquence immédiate du théorème de Skolem-Mahler-Lech, voir le § II ).

Une étude du cas des suites récurrents linéaires à valeurs complexes est faite par Beukers et Tijdeman en [ B.T. ], à ce sujet voir aussi les notes du chapitre 4 de [ Sh.T.] . 


\section{Minoration de $P\left(u_{n}\right)$.}

Dans tout ce paragraphe, on considère une suite récurrente linéaire $\left(\mathrm{u}_{n}\right)$ à valeurs entières telle qu'aucun des quotients $\omega_{\mathrm{i}} / \omega_{\mathrm{j}}, \mathrm{i} \neq \mathrm{j}$, ne soit une racine de l'unité et qui possède au moins deux racines $\omega_{\mathrm{i}}$ distinctes ( autrement dit, on a $k \geq 2$ ). On s'intéresse à $P\left(u_{n}\right)$, le plus grand diviseur premier de $u_{n}$ (par convention : $P(0)=P( \pm 1)=1$ ).

Dès $1921, G$. Pólya a montré que la limite supérieure de $P\left(u_{n}\right)$ est infinie (l'exemple de la suite $u_{n}=n^{h} 2^{n}$, pour laquelle on a $P\left(u_{2^{m}}\right)=2$ pour tout $m$ positif, montre que l'hypothèse $\mathrm{k} \geq 2$ est nécessaire ). En 1934, K. Mahler [ Ma1 ] a démontré que $P\left(u_{n}\right)$ tend en fait vers l'infini lorsque $\left(u_{n}\right)$ est binaire, ceci en utilisant une généralisation p-adique du théorème de Thue-Siegel, résultat rendu effectif par Schinzel en 1967 , grâce à la théorie de Gel'fond, voir [ Schi ] .

En utilisant le théorème fondamental des S-unités, R. van der Poorten et H. P. Schlikewei ont montré que s'il y a au moins deux racines $\omega_{\mathrm{i}}$ distinctes alors le nombre premier $P\left(u_{n} /\right.$ p.g.c.d. $\left.\left(u_{m}, u_{n}\right)\right)$ tend en fait vers l'infini si $m \rightarrow \infty, m>n$ et $u_{n} \neq 0$; une preuve indépendante mais voisine a aussi été donnée par J. Evertse [ Ev ] . A cc jour, ces résultats ne sont pas effectifs.

Par contre, comme dans le cas de la minoration de $\left|u_{n}\right|$, la théorie de Baker permet dans certains cas d'obtenir une minoration effective de $P\left(u_{n}\right)$. Ici, on a besoin qu'il y ait une seule racine dominante (i. e. de la condition $\left|\omega_{1}\right|>\left|\omega_{2}\right| \geq \ldots$ ), voir le résultat de C. Stewart publié en [ Ste2 ]. Quand elle s'applique, une telle estimation fournit évidemment un algorithme permettant (en principe) de résoudre les inéquations de la forme $P\left(u_{n}\right) \leq k$, où $k$ est un entier fixé. Un tel algorithme a été mis en oeuvre par B. de Weger, voir [ W ] . En fait, en pratique, de telles inéquations sont souvent résolues assez rapidement de manière élémentaire : on considère la suite $\left(u_{n}\right)$ modulo certains nombres premiers convenablement choisis (des exemples figurent en [ Stø] , [Mi4 ] , [Mi5 ] , $[\mathrm{Mi6}],[\mathrm{Mu}], \ldots)$. 
Dans le cas d'une suite récurrente linéaire à valeurs entières qui possède deux racines dominantes, il ne semble pas que l'on connaisse un algorithme permettant de résoudre les inéquations de la forme $P\left(u_{n}\right) \leq k$, où $k$ est un entier fixé. D'où l'intérêt d'essayer de résoudre une telle inéquation dans certains cas particuliers. Le cas de la suite de Berstel $\left(b_{n}\right)$ et de l'inéquation $P\left(b_{n}\right) \leq 3$ a été traité de manière élémentaire en [M.T. ], où on montre qu'il y a 44 solutions $n \in Z$.

On peut aussi démontrer des résultats sur le nombre $Q\left(u_{n}\right)$, où $Q\left(u_{n}\right)$ désigne le plus grand diviscur quadratfrei de $u_{n}$. C'est ainsi que C. L. Stewart [Ste1] a démontré que si $a, b, x, y$ sont des entiers non nuls, avec $x \neq y$, alors on peut effectivement déterminer des nombres $\mathrm{C}$ et $\mathrm{N}$, qui ne dépendent que de $\mathrm{a}$ et $\mathrm{b}$, tels l'on ait

$$
Q\left(a x^{n}+b y^{n}\right) \geq C \frac{m}{(\log m)^{2}} \text { pour } n \geq N \text {. }
$$




\section{VI . Etude des répétitions .}

Parmani et Shorey [P.Sh.] ont démontré le résultat suivant: si $\left(u_{n}\right)$ est une suite binaire non dégénérée à valeurs algébriques avec deux racines $\omega_{1}$ et $\omega_{2}$ telles que le quotient $\omega_{1} / \omega_{2}$ ne soit pas une racine de l'unité alors il existe une constante calculable $\mathrm{C}$ telle que l'on ait $u_{m} \neq u_{n}$ pour $\max \{m, n\}>C$.

Puis Shorey [ Sho2 ] a obtenu un résultat plus précis, il minore la distance entre deux termes $u_{m}$ et $v_{n}$ d'une suite récurrente binaire : sous les mêmes hypothèses que le résultat précédent, avec de plus $\left|\omega_{1}\right| \geq\left|\omega_{2}\right|$, on a la minoration

$$
\left|u_{m}-u_{n}\right| \geq\left|\omega_{1}\right| \max (m, n\} .(m+2)-C \log (n+2)
$$

si $m \neq n$ et $\max \{m, n\} \geq C^{\prime \prime}$.

Lewis et Turk [ L.T. ] considèrent l'équation $u_{m}=a u_{n}$ pour a nombre fixé et $u_{n}$ binaire. Ils démontrent aussi que si on se donne des entiers $m>n>p>q$, il n'y $a-a$ multiplication près par une constante - qu'un nombre fini de suites récurrentes linéaires non dégénérées qui vérifient $u_{m}=u_{n}=u_{p}=u_{q}$ et que l'on peut prendre ces suites à valeurs algébriques. Cette question est traitée dans le cas général par M. Laurent dans [ Lau2 ] .

Dès 1979, on trouve en [Mi3] une étude de l'équation $u_{m}=v_{n}$ où $\left(u_{m}\right)$ et $\left(v_{n}\right)$ sont des suites récurrentes linéaires à valeurs algébriques possédant chacune une seule racine dominante. Là encore, le cas général a été traité par M. Laurent . Grâce au théorème des sous-espaces de Schmidt - Schlickewei, il démontre le résultat remarquable suivant (voir [ Lau2 ], th. 3):

Soient $u_{n}=\sum_{i=1}^{h} f_{i}(n) \alpha_{i} n \quad$ et si $\quad v_{n}=\sum_{j=1}^{k} g_{i}(n) \beta_{j} n \quad$ deux suites récurrentes linéaires non - dégérées de nombres algébriques, avec $h \geq 2$ et $k \geq 2$. Alors, si l'équation $u_{m}=v_{n}$ admet une infinité de solutions les propriétés suivantes ont lieu,

$1^{\circ}$ ) on a $h=k, \quad 2^{\circ}$ ) il existe des entiers strictement positifs $r$ et $s$, tels qu'après réindexation éventuelle des nombres $\beta_{j}$ on ait les relations $\alpha_{i}{ }^{r}=\beta_{i}{ }^{3}$ pour $1 \leq i \leq h$, $3^{\circ}$ ) à un nombre fini d'exceptions près, la condition $u_{m}=v_{n}$ implique les relations

$$
f_{i}(n) \alpha_{i} n=g_{i}(n) \beta_{i} n \text { pour } \quad 1 \leq i \leq h \text {. }
$$


VII . Questions de rationalité .

Les problèmes abordés dans ce paragraphe ne sont pas traités en [ C.M.P.] , pour plus de détails nous renvoyons le lecteur à l'article de van der Poorten [ Poo2 ] .

Le point de départ de ce paragraphe est le théorème de Hadamard : si $\left(u_{n}\right)$ et $\left(v_{n}\right)$ sont deux suites récurrentes linéaires alors leur produit de Hadamard $\left(u_{n} \cdot v_{n}\right)$ est encore une série rationnelle ; théorème qui résulte immédiatement de la formule (5), puisque le produit de deux polynômes exponentiels est encore clairement un polynôme exponentiel. Beaucoup de travaux portent sur diverses réciproques possibles de ce théorème ; dans ce but, il est nécessaire de posséder des critères de rationalité.

Un des premiers critères de rationalité est celui de Hankel : Soit $\mathbf{u}=\left(\mathrm{u}_{\mathrm{n}}\right)$ une suite à valcurs dans un corps, pour $n$ et $s$ entiers on définit le déterminant de Hankel $D_{n}^{s}(u)$ :

$$
D_{n}^{s}(u)=\operatorname{det}\left(\begin{array}{ccc}
u_{n} & \ldots & u_{n+s} \\
& \ldots & u_{n+2 s} \\
u_{n+s} & \ldots & u_{n+2}
\end{array}\right) ;
$$

la suite $\mathbf{u}$ est une suite récurrente linéaire si, et seulement si, le déterminant $D_{\mathbf{n}}^{\mathbf{s}}(\mathbf{u})$ est nul pour tout $\mathrm{n}$ assez grand.

Il est clair que cette condition est nécessaire. La réciproque résulte aisément de la formule

$$
D_{n}^{m} \quad D_{n+2}^{m-2}=D_{n+2}^{m-1} D_{n}^{s} \cdot D_{n}^{m-1}-\left(D_{n}^{s}\right)^{2},
$$

elle-même conséquence de la relation de Sylvester ( voir [ Pi ] , pp. 21 - 22) .

Le critère de Hankel implique presque directement un autre critère, dû à Kronecker : la suite u est une suite récurrente linéaire si, et seulement si, le déterminant $D \mathfrak{g}$ pour tout $n$ assez grand ( voir [ Pi ] , pp. $24-25$ ).

On trouvera d'autres critères de rationalité en [ $\mathrm{Pi}]$ et [ Poo2 ], $\$ 4.4$, ainsi que des résultats fondamentaux de J.F. Ritt [Ri1] et [Ri2 ]. 
Un des premiers résultats de rationalité est le lemme de Pólya - Cantor : soit $\sum_{n} u_{n} X^{n}$ une série formelle à coefficients entiers algébriques, appartenant à un corps de nombres fixe, et soit $P$ un polynôme non nul, alors, si la sćrie $\sum_{n \geq 0} P(n) u_{n} X^{n}$ est rationnelle, il en est de même de la série $\sum_{n} u_{n} X^{n}$. Le cas particulier de ce résultat, où $P(n)=n$, est dû à Pólya , [ Pó1 ] , il a été ensuite généralisé par Cantor , [ Can1 ] .

Le lemme de Pólya - Cantor constitue le premier pas vers le théorème dit théorème des quotients de Hadamard : Soit $\mathrm{K}$ un corps de caractéristique nulle et soit $\left(\mathrm{u}_{\mathrm{n}}{ }^{\prime}\right)$ une suite d'éléments d'un sous-anneau $R$ de $K$, qui est de type fini sur $Z$; soient $\left(v_{n}\right)$ et $\left(w_{n}\right)$ deux suites récurrentes linéaires à valeurs dans $K$; on suppose que l'on a $u_{n}{ }^{\prime}=v_{n} / w_{n}$ pour tout $n$ tel que $w_{n} \neq 0$. Alors, il existe une suite récurrente linéaire $\left(u_{n}\right)$ qui vérific $u_{n}=u_{n}{ }^{\prime}$ pour tout $n$ tel que $w_{n} \neq 0$.

Dans le cas où les suites sont à valeurs entières, ce résultat a été conjecturé par Pisot (conjecture citcé dans l'appendice de la thèse de Benzaghou [ Ben ] ). Le cas particulier où $\mathrm{w}_{\mathrm{n}}$ ne possède qu'une racine de plus grand module a été démontré par Cantor [ Can2 ] . Le résultat a été annoncé par Pourchet [ Po ], mais sa preuve ne semble pas complète. Il a été complètement démontré par van der Poorten [ Poo1], voir aussi la présentation détaillée de la preuve de van der Poorten donnée par Rumely [ Rum] .

Ces questions sont aussi liées à des théorèmes de Borel - Pólya - Carlson, généralisés par B. Dwork, A.M. Bertrandias, D. Cantor. Voir les références [ Bert1 ], [ Bert2 ], [Bo ], [ Pó2 ], [ Dw ], [ Can2] . 


\section{Bibliographie}

La bibliographie qui suit comporte surtout des travaux assez récents, mais elle est certainement très incomplète. Quelques uns des articles qui y figurent ne sont pas référencés dans le texte, ceci du fait qu'ils traitent de questions qui n'ont pas été abordées dans notre étude.

[A] Y. Amice . - Les nombres p-adiques; Paris, P.U.F., 1975.

[ Bac ] P. Bachmann .-Niedere Zahlentheorie, Zweiter Teil, Leipzig, Teubner, 1910.

[ Bak ] A. Baker . - The theory of linear forms in logarithms ; Transcendence Theory : Advances and Applications, Academic Press, London, 1977, pp. 1 - 27.

[Ben ] B. Benzaghou . - Algèbres de Hadamard ; Bull. Soc. Math. de France, v. 98, 1970, pp. $209-252$.

[ Berl] J. Berstel . - Transductions and context-free languages ; Stuttgart, Teubner, 1979.

[ Ber2] J. Berstel . - Factorisation des fractions rationnelles et suites récurrentes ; Acta Arith. , v. 30, 1976, pp. $365-376$.

[Bert 1] F. Bertrandias . - Diamètre transfini dans un corps valué. Application au prolongement analytique ; C. R. Acad. Sci. Paris , v. 257,1963 , pp. 583 - 585.

[ Bert 2] F. Bertrandias - - Séries de Taylor à coefficients rationnels ; C. R. Acad. Sci. Paris , v. 257,1963 , pp. 3793 - 3795 .

[ B.M. ] J. Berstel, M. Mignotte . - Deux propriétés décidables des suites récurrentes linéaires ; Bull. Soc. Math. France , v. 104, 1976, pp. 175 - 184.

[ B.R. ] J. Berstel, Ph. Reutenauer . - Les séries rationnelles et leurs langages; Paris, Masson, 1984.

[ Beu1] F. Beukers . - The multiplicity of binary recurrences ; Compositio Math. , v. 40, 1980, pp. $251-267$.

[Beu2] F. Beukers . - The zero-multiplicity of ternary recurrences; 1982, unpublished.

[ Bez1] J. P. Bézivin . - Suites récurrentes et polynômes à plusieures variables ; Groupe d'étude d'analyse ultramétrique, $1982 / 83, \mathrm{n}^{\circ} 2,6$ pages. 
[ Bez1 ] J. P. Bézivin . - Suites récurrentes et polynômes à plusieures variables

[ Bez2 ] J. P. Bézivin - - Factorisation des suites récurrentes linéaires et applications ; Bull. Soc. Math. France, v. 112, 1984, pp. 365 - 376.

[ Bez3 ] J. P. Bézivin . - Une généralisation du théorème de Skolem-Mahler; manuscrit .

[ Bez4 ] J. P. Bézivin . - Sur un théorème de Pólya ; J. für die reine und angewandte Math., v. 364,1986 , pp. $60-68$.

[ Bez5] J. P. Bézivin . - Sur les diviseurs premiers des suites récurrentes linéaires; Ann. Fac. Sci. de Toulouse ; v. 8, $\mathrm{N}^{\circ} 1,1986 / 87$, pp. $61-73$.

[ B.Ro.] J. P. Bézivin, Ph. Robba - - Rational solutions of linear differential equations ; $J$. Austral. Math. Soc. , v. $44,1988$.

[ Bo ] E. Borel . - Sur une application d'un théorème de M. Hadamard ; Bull. Sci. Math. ( $2^{\text {ème }}$ ser. ), v. 18,1894 ,pp. $22-25$.

[ B.S.] Z. I. Borevich, I. R. Shafarevich . - Number Theory ( russian ). English edn, Pure Apl. Math. , v. 20, Academic Press, New York, 1966.

[ B.T.] F. Beukers, R. Tijdeman . - On the multiplicity of binary complex sequences ; Compositio Math. , v. 51 , 1984, pp. 193 - 213.

[ Can1] D. G. Cantor . - On arithmetic properties of the Taylor series of rational functions ; Canadian J. Math. , v. 21, 1969, pp. 378 - 382 .

[ Can2] D. G. Cantor . - On arithmetic properties of the Taylor series of rational functions II ; Pacific J. Math. , v. 41, 1972, pp. 329 - 334 .

[ Can3] D. G. Cantor . - On an extension of the definition of transfinite diameter and some applications ; J. für die reine und angew. Math. , v. 316 , 1980 , pp. 160 - 207.

[ Car ] R. D. Carmichael . - On sequences of integers defined by recurrence relations ; Quaterly J. Math., v. 48, 1920, pp. 343 - 372 .

[ Cas ] J. W. S. Cassels . - Local fields ; London Math. Soc. Stud. Texts 3 , Cambridge, Cambr. Univ. Press, 1986.

[ C.M.P. ] L. Cerlienco, M. Mignotte, F. Piras . - Suites récurrentes linéaires, Propriétés algébriques et arithmétiques; L'Enseign. Math. , t. 33, 1987, pp. $67-108$. 
[ Co ] L. Comtet , - Analyse Combinatoire ; Paris, P.U.F., 1970.

[Des ] B. Deshommes . - Sur les zéros des fonctions symétriques complètes des corps cubiques ; Pacific J. Math. , à paraître.

[ Dia ] A. Diab . - Sur les zéros communs des polynômes exponentiels : C. R. Acad. Sci. Paris , v. 281,1975 , Ser. A , pp. $757-758$.

[Di] L.E. Dickson . - History of the theory of numbers ; 3 v. , New York, Chelsea, 1952 .

[ Dw ] B. Dwork - - On the rationality of the zeta-function of an algebraic variety; Amer. J. Math. , v. 82,1960 , pp. $631-648$.

[ E.G.S.T. ] J. H. Everste, K. Györy, C. Stewart, R. Tijdeman . - S - units equations and their applications ; Report W 87 - 03 , Mathematical Inst. , Univ. Leiden , jan. 87 ; to appear in A. Baker, Proceedings of the Durham Symposium on Transcendence Theory, 1986.

[ Ev ] J. H. Evertse . - On sums of S-units and linear recurrences ; Compositio Math., v. 53 , pp. $225-244$

[Fa ] P. Fatou . - Séries trigonométriques et séries de Taylor ; Acta Math. , v. 30, 1906, pp. $335-400$.

[ Fi ] L. Fibonacci .- Liber Abaci.; 1202 .

[ Ge ] Gel'fond - - Calcul des différences finies; Paris, Dunod, 1963.

[ G.L.P.1] J. P. Glass, J. H. Loxton, A. J. van der Poorten . - Identifying a rational function; C. R. Math. Rep. Acad. Sci. Canada , v. 7, 1981, pp. 279 - 284 .

[ G.L.P.2] J. P. Glass, J. H. Loxton, A. J. van der Poorten .- On the total multiplicity of recurrence sequences; to appear .

[ Go ] S. W. Golomb . - Shift register sequences; San Francisco, Holden-Day, 1967.

[ Gy ] K. Györy . - On some arithmetic properties of Lucas and Lehmer numbers; Acta Arith. , v. 40, pp. $369-373$.

[ G.K.S. ] K. Györy, P. Kiss, A. Schinzel . - On Lucas and Lehmer sequences and their applications to diophantine equations ; Colloq. Math. , v. 45, 1981, pp. 75 - 80. 
[ He ] P. Henrici . - Elements of numerical Analysis; New York, Wiley, 1964 .

[ Ki 1] P. Kiss . - Zero terms in second order linear recurrences; Math. Sem. Notes Kobe Univ. , ( Japan, ), v. 7, 1979, pp. 145 - 152 .

[ Ki 2] P. Kiss . - On common terms of linear recurrences ; Acta Math. Acad. Sci. Hung., v. 40,1982 , pp. $119-123$.

[Ki 3] P. Kiss . - Differences of the terms de linear recurrences; Studia Sci. Math. Hung., 1985, v. 20, pp. $285-293$.

[ Kn ] D. E. Knuth . - The art of computer programming , v. 2, semi-numerical algorithms; New-York, Addison-Wesley, 1981.

[ Ku ] K. Kubota . - On a conjecture of M. Ward I, II, III ; Acta Arith. , v. 33, 1977, pp. $11-28,29-48$, pp. $99-109$.

[ L. W. ] J. C. Lagarias, D. P. Weisser . - Fibonacci and Lucas cubes ; Fibonacci Quart. , v. 19,1981 , pp. $39-43$.

[ Las ] A. Lascoux . - Suites récurrentes linéaires ; Advances in applied math. , v. 7 , 1986, pp. $228-235$.

[Lau1] M. Laurent .- Equations diophantiennes exponentielles ; Invent. Math.,1984, v. 78, pp. $299-327$.

[Lau2] M. Laurent - Equations exponentielles polynômes et suites récurrentes linéaires ; Journées Arithmétiques de Besançon, juin 1985 , Astérisque, $\mathrm{n}^{\circ} 147$ - 148 , 1987, pp. $121-139$.

[Le] C. Lech . - A note on recurring sequences; Ark. Mat., v. 2, 1953, pp. 417 - 421.

[Lev ] W. LeVeque . - Topics in Number Theory, v. 1, Addison-Wesley, Reading, Mass., 1958.

[Lew ] D. J. Lewis . - Diophantine equations : p-adic methods . Studies in Number Theory 6, ed. W. J. LeVeque, Englewoods Cliffs, New Jersey, 1969.

[ L.M. ] D. J. Lewis, P. Morton . - Quotients of polynomials and a theorem of Pisot. - $J$. Fac. Sci. of Tokyo Univ. , v. 28, $\mathrm{n}^{\circ} 3,1981$, pp. $813-823$. 
[ L.N. ] R. Lidl, H. Niederreiter . - Introduction to finite fields and their applications; Cambridge, Cambr. Univ. Press, 1986.

[ L. P. ] J. H. Loxton, A. J. van der Poorten . - On the growth of recurrence sequences ; Math. Proc. Camb. Phil. Soc., 1977, v. 81, pp. 369 - 376.

[ L. T. ] D. J. Lewis , J. Turk . - Repetitiveness in binary sequences ; J. für die reine angew.Math. , 1985, v. 356, pp. $19-48$.

[ Lu ] E. Lucas . - Théorie des nombres ; Paris, Gauthier-Villars, 1891.

[ McW.Sl. ] F. J. MacWilliams, N. J. A. Sloane . - The theory of error correcting codes ; Amsterdam, North-Holland, 1978 .

[Ma1] K. Mahler . - Eine arithmetische Eigenshaft der rekurrierenden Reihen ; Mathematica B, Leiden, 1934, v. 3, pp. $153-156$.

[ Ma2 ] K. Mahler - Eine arithmetische Eigenschaft der Taylor-Koeffizienten rationaler Funktionen ; Proc. Akad. Wetensch. Amsterdam , v. 38, 1935, pp. 50 - 60 .

[ Ma3 ] K. Mahler . - A remark on linear recurrent sequences ; J. Math. Sci. , v. 1, 1966, pp. $12-17$.

[Ma4] K. Mahler . - Introduction to p-adic numbers and their functions, Cambridge, Camb. Univ. Press, 1973.

[ Mac ] I. G. Macdonald - - Symmetric functions and Hall polynomials; Oxford Univ. Press, 1979 .

[ Mi1] M. Mignotte - Suites récurrentes linéaires ; Sém. Delange - Pisot - Poitou, v. 15 , $1973 / 74$, Gr. ét. nº 14,9 pages.

[ Mi 2 ] M. Mignotte . - A note on linear recurrent sequences ; J. Austral. Math. Soc., Ser $A$, v. 20,1975 , pp. $242-244$.

[ Mi 3 ] M. Mignotte - - Une extension du théorème de Skolem - Mahler ; C. R. Acad. Sci. , Paris , ser. A , 1979, v. 288, pp. 233 - 235.

[Mi 4] M. Mignotte . - On the automatic resolution of certain diophantine equations ; Proc. de EUROSAM 84 , Lecture Notes in Computer Science 174, Springer-Verlag, Berlin, 1984, pp. 378 - 385 . 
[Mi 5] M. Mignotte . - Une nouvelle résolution de l'équation $x^{2}+7=2^{n} ;$ Rend. del Sem. Univ. Cagliari, v. 54, n 2, 1984, p. $41-43$.

[ Mi 6] M. Mignotte .- $\mathrm{P}\left(\mathrm{x}^{2}+1\right) \geq 17$ si $\mathrm{x} \geq 240 ;$ C. R. Acad. Sci. Paris , t. 301, serie $I, n^{\circ} 13,1985$, p. $661-664$.

[ Mi 7] M. Mignotte - - Détermination des répétitions d'une certaine suite récurrente linéaire, Acta Math. Debrecen , t. 33, 1986 , p. 297 - 306 .

[ M.S.T. ] M. Mignotte, T. Shorey, R. Tijdeman .- The distance between terms of an algebraic recurrence sequence ; J. für die reine angew. Math. , v. 349, 1984, pp. 63 - 76.

[ M-Th. ] L. M. Milne-Thomson . - The calculus of finite differences; London, MacMillan, 1960 .

[Mo] P. Montel . - Leçons sur les récurrences et leurs applications ; Paris, Gauthier-Villars, 1957.

[ $\mathrm{Mu}$ ] M. Mureddu . - A lower bound for $\mathrm{P}\left(\mathrm{x}^{4}+1\right)$; Ann. Fac. Sci. Toulouse, v. $8, n^{\circ} 2,1986 / 87$, pp. $109-119$.

[ Na ] T. Nagell . - Introduction to Number Theory, Chelsea, New York, 1964.

[ N.P.1] I. Nemes, A. Pethö . - Polynomial values in linear recurrences ; Publ. Math. Debrecen, 1984, v. 31, pp. 229 - 233 .

[ N.P.2] I. Nemes, A. Pethö . - Polynomial values in linear recurrences II ; J. Number Th. , v. 24,1986, pp. $47-53$.

[Mo] P. Montel . - Leçons sur les récurrences et leurs applications ; Paris, Gauthier-Villars, 1957.

[ $\mathrm{Pa}$ ] G. Pathiaux . - Algèbre de Hadamard de fractions rationnelles; C. R. Acad. Sci. Paris , v. 267, 1968, Ser. A, pp. $977-979$.

[ Pe1] A. Pethö . - Perfect powers in second order linear recurrences ; J. Number Theory, 1982, v. 15, pp. $5-13$.

[ Pe 2] A. Pethö . - Full cubes in the Fibonacci sequence; Publ. Math. Debrecen, 1983, v. 30 , pp. $117-127$. 
[ Pe3] A. Pethö . - Perfect powers in second order recurrences; Topics in classical Number Theory , Proc. Conf. Budapest, 1981, Colloq. Math. Soc. János Bolyai, v. 34, North-Holland, Amsterdam, pp. 1217 - 1227.

[Pe4] A. Pethö . - On the solution of the diophantine equation $\mathrm{G}_{n}=\mathrm{p}^{2}$; Proc. EUROCAL'85, Linz, Lecture Notes in Computer Science 204, Springer-Verlag, Berlin, pp. $503-512$.

[ Pi ] C. Pisot . - Quelques aspects de la théorie des entiers algébriques ; Sém. Univ. Montréal, 1963.

[Pic ] P. A. Picon - - Sur certaines suites récurrentes cubiques ayant deux ou trois termes nuls ; Discrete Math. , v? 21, 1978, pp. 285 - 296.

[Po ] Y. Pourchet . - Solution du problème arithmétique du quotient de Hadamard de deux séries rationnelles ; C.R. Acad. Sci.Paris , v. 288, 1979, Ser. A, pp. 1055 - 1057.

[ Pó1 ] G. Pólya . - Arithmetisches Eigenschaften der Reihenentwicklungen rationaler Funktionen, J. für die reine angew. Math. , 1921, v. 151, pp. 1 - 31 .

[Pó2] G. Pólya . - ; Über qewisse notwendige Determinantenkriterien für der Fortsetzbarkeit einer Potenzreihe; Math. Ann. , v. 99, 1928 , pp. $687-706$.

[P.S.] G. Pólya, G. Szegö . - Problems and theorems in analysis ; Berlin, Springer-Verlag, 4 ième édition, traduction anglaise, 1976.

[ Poo1 ] A. J. van der Poorten . - Solution de la conjecture de Pisot sur le quotient de Hadamard de deux fractions rationnelles ; C.R. Acad. Sci. Paris , v. 306, série 1, 1988, pp. 97 - 102 .

[ Poo2 ] A. J. van der Poorten . - Some facts that should be better known, especially about rational functions ; Macquarie Mathematical reports , $n^{\circ}$ 88-0022, juin 1988 (à paraître dans Number Theory and Applications, ed. R. A. Mollin , D. Reidel Publ. Co. , Dordrecht, 1989 ).

[ Poo3 ] A. J. van der Poorten . - P - adic metods in the study of Taylor coefficients of rational functions; Bull. Austral. Math. Soc. , v. $28,1984$.

[ P.R. ] A. J. van der Poorten, R. S. Rumely . - Zeros of p-adic exponential polynomials II ; J. London Math. Soc. , v. 36, 1987, pp. 1 - 15. 
[ P.Sch.] A. J. van der Poorten, H. P. Schlickewei .- The growth condition for recurrence sequences, Report 82-0041, Macquarie University, N.S.W., Australia, 1982.

[ P. Sh. ] J. C. Parmani, T. N. Shorey .- Subsequences of binary recurrent sequences ; Acta Arith. , v. 40, 1982, pp. $193-196$.

[ Re ] C. Reutenauer. - Sur les éléments inversibles de l'algèbre de Hadamard des séries rationnelles ; Bull. Soc. Math. France, v. 110, 1982, pp. 225 - 233.

[Ri1] J. F. Ritt - - A factorisation theory for functions $\sum_{i=1}^{n} a_{i} e^{\alpha_{i} z} ;$ Trans. Amer. Math. Soc. , v. 29, 1927, pp. $584-596$.

[Ri2 ] J. F. Ritt . - Algebraic combinations of exponentials ; Trans. Amer. Math. Soc., v. 31,1927 , pp. $654-679$.

[ Ro] Ph. Robba . - Zéros de suites récurrentes linéaires; Groupe d'étude d'analyse ultramétrique , $1977 / 78$, Paris, exp. $n^{\circ} 13,5$ pp.

[ Rue ] R. A. Rueppel - - Analysis and design of stream ciphers; Berlin, Springer-Verlag, 1986.

[Rum ] R. S. Rumely . - Notes on van der Poorten's proof of the Hadamard quotient theorem ; Séminaire de Théorie des Nombres de Paris , 1986/87 , Birkaüser, 1988.

[ R.vdP.1 ] R. S. Rumely . and A. J. van der Poorten - - Remarks on generalized power sums ; Bull. Austral. Math. Soc. , v. 36, 1987 , pp. 311 - 329 .

[ R.vdP.2 ] R. S. Rumely . and A. J. van der Poorten . - A note on the Hadamard $k^{\text {th }}$ root of a rational fuction; J. Austral. Math. Soc. , Ser. A , v. 43, 1987 , pp. 314 - 327 .

[ Sc] S. J. Scott . - On the number of zeroes of a cubic recurrencee ; Am. Math. Monthly , v. 67,1960 ,pp. $169-170$.

[ Schi ] A. Schinzel . - On two theorems de Gel'fond and some of their applications; Acta Arith. , v. 13, 1967, pp. $177-236$.

[ Se ] E. S. Selmer . - Linear recurrence relations over finite fields; Univ. of Bergen, Dept. of Math. , Norway, 1966.

[ Sho1] T. N. Shorey . - The greatest square-free factor of a binary recurrent sequence ; Hardy - Ramanujan J. , 1983, v. 6, pp. 23 - 36. 
[ Sho2 ] T. N. Shorey . - Linear forms in members of a binary recursive sequence; Acta Arith. , v. 43,1984 , pp. $317-331$.

[ Sh.T ] T. N. Shorey, R. Tijdeman .- Exponential diophantine equations ; Cambridge Tracts in Math. 87,1986 , Cambridge Univ. Press .

[Sk] Th. Skolem - - Ein Verfahren zur Behandlung gewisser exponentialer Gleichlungen und diophantischer Gleichlungen , 8. Skand. Mat. Kong., Stockholm , 1934 , pp. $163-188$.

[Sm] M. F. Smiley . - On the zeroes of a cubic sequence; Am. Math. Monthly, v. 63 , 1956 , pp. $171-172$.

[Ste1] C. L. Stewart . - On divisors of terms de linear recurrence sequences ; J. für die reine angew. Math. , v. 333, 1982, pp. 12 - 31 .

[Ste2] C. L. Stewart . - On the greatest prime factor of terms of a linear recurrence sequence; Rocky Mountain J. Math., v. 15, n², pp. 599 - 608.

[Stø] C. Størmer . - Quelques théorèmes sur l'équation de Pell $\mathrm{x}^{2}-\mathrm{D} \mathrm{y}^{2}= \pm 1$ et leurs applications, Vid. - Selsk. Skrifter. Math. Naturv. Kl, (Christiana ) , 1897, n 2,48 pp.

[ Str ] R. Strassmann . - Uber den Wertevorrt von Potenzreihen im Gebeit $\wp$-adischen Zahlen ; J. für die reine und angew. Math. , v. 159,1928 , pp. $19-28$ \& pp. 65 - 66 .

[ Ti ] R. Tijdeman . - Multiplicities of binary recurrent sequences; Sém. Théorie des nombres, Bordeaux, $1980 / 81, \mathrm{n}^{\circ} 19,11$ pages.

[U] S. Uchiyama .- On a theorem of G. Pólya ; Proc. Japan Acad., v. 41, 1969 , pp. $517-520$.

[V] M. Voorhoeve . - Zeroes of exponential polynomials, Thesis, Leiden, 1977.

[W] B. de Weger - - Solving exponential diophantine equations using lattice basis reduction algorithms; J. Number Theory , 26, 1987, pp. $325-367$.

[Z] N. Zierler - - Linear recurring sequences and error-correcting codes; in Error correcting codes, ed. H. B. Mann, Wiley, New-York, 1968, pp. 47 - 59.

\author{
M. Mignotte \\ Mathématique, Université Louis Pasteur \\ 67084 Strasbourg, France
}

